\title{
Engineering a Simple, Efficient Code-Generator Generator
}

CHRISTOPHER W. FRASER

AT \& T Bell Laboratories

DAVID R. HANSON

Princeton University

and

TODD A. PROEBSTING

The University of Arizona

Many code-generator generators use tree pattern matching and dynamic programming. This paper describes a simple program that generates matchers that are fast, compact, and easy to understand. It is simpler than common alternatives: 200-700 lines of Icon or 950 lines of $\mathrm{C}$ versus 3000 lines of $\mathrm{C}$ for Twig and 5000 for burg. Its matchers run up to 25 times faster than Twig's. They are necessarily slower than burg's BURS (bottom-up rewrite system) matchers, but they are more flexible and still practical.

Categories and Subject Descriptors: D.3.4 [Programming Languages]: Processors-code generation; compilers; translator writing systems and compiler generators

General Terms: Languages

Additional Key Words and Phrases: Code generation, code-generator generator, dynamic programming, Icon programming language, tree pattern matching

\section{INTRODUCTION}

Many code-generator generators use tree pattern matching and dynamic programming (DP) $[2,4,8]$. They accept tree patterns and associated costs, and semantic actions that, for example, allocate registers and emit object code. They produce tree matchers that make two passes over each subject tree. The first pass is bottom up and finds a set of patterns that cover the tree with minimum cost. The second pass executes the semantic actions associated with minimum-cost patterns at the nodes they matched. Code-generator generators based on this model include BEG [7], Twig [3], and burg [13].

Authors' addresses: C. W. Fraser, AT \& T Bell Laboratories, 600 Mountain Avenue 2C-464, Murray Hill, NJ 07974-0636; D. R. Hanson, Department of Computer Science, Princeton University, Princeton, NJ 08544; T. A. Proebsting, Department of Computer Science, The University of Arizona, Tucson, AZ 85721.

Permission to copy without fee all or part of this material is granted provided that the copies are not made or distributed for direct commercial advantage, the ACM copyright notice and the title of the publication and its date appear, and notice is given that copying is by permission of the Association for Computing Machinery. To copy otherwise, or to republish, requires a fee and/or specific permission.

(C) 1992 ACM 1057-4514/92/0900-0213 $\$ 01.50$

ACM Letters on Programming Languages and Systems, Vol. 1, No. 3, September 1992, Pages $213-226$. 
BEG matchers are hard-coded and mirror the tree patterns in the same way that recursive-descent parsers mirror their input grammars. They use $\mathrm{DP}$ at compile time to identify a minimum-cost cover.

Twig matchers use a table-driven variant of string matching $[1,15]$ that, in essence, identifies all possible matches at the same time. This algorithm is asymptotically better than trying each possible match one at a time, but overhead is higher. Like BEG matchers, Twig matchers use DP at compile time to identify a minimum-cost cover.

burg uses BURS (bottom-up rewrite system) theory $[5,6,17,18]$ to move the DP to compile-compile time. BURS table generation is more complicated, but BURS matchers generate optimal code in constant time per node. The main disadvantage of BURS is that costs must be constants; systems that delay DP until compile time permit costs to involve arbitrary computations.

This paper describes a program called iburg that reads a burg specification and writes a matcher that does DP at compile time. The matcher is hard-coded, a technique that has proved effective with other types of code generators $[9,12]$. iburg was built to test early versions of what evolved into burg's specification language and interface, but it is useful in its own right because it is simpler and thus easier for novices to understand, because it allows dynamic cost computation, and because it admits a larger class of tree grammars [16]. iburg has been used with good results in a first course on compilers. burg and iburg have been used also to produce robust VAX, MIPS, and SPARC code generators for lcc, a retargetable compiler for ANSI $\mathrm{C}$ [11].

iburg and BEG produce similar matchers, but this paper describes them in more detail than the standard BEG reference [7]. In particular, it describes several optimizations that paid off and two that did not, and it quantifies the strengths and weaknesses of such programs when compared with programs like Twig and burg.

\section{SPECIFICATIONS}

Figure 1 shows an extended BNF grammar for burg and iburg specifications. Grammar symbols are displayed in italic type, and terminal symbols are displayed in typewriter type. $\{X\}$ denotes zero or more instances of $X$, and $[X]$ denotes an optional $X$. Specifications consist of declarations, a $\% \%$ separator, and rules. The declarations declare terminals - the operators in subject trees-and associate a unique, positive external symbol number with each one. Nonterminals are declared by their presence on the left side of rules. The \%start declaration optionally declares a nonterminal as the start symbol. In Figure 1 term and nonterm denote identifiers that are terminals and nonterminals, respectively.

Rules define tree patterns in a fully parenthesized prefix form. Every nonterminal denotes a tree. Each operator has a fixed arity, which is inferred from the rules in which it is used. A chain rule is a rule whose pattern is another nonterminal. If no start symbol is declared, the nonterminal defined by the first rule is used. 


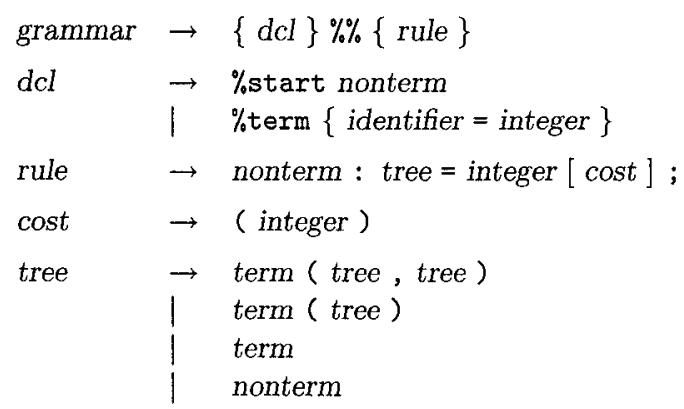

Fig. 1. Extended BNF grammar for burg and iburg specifications.

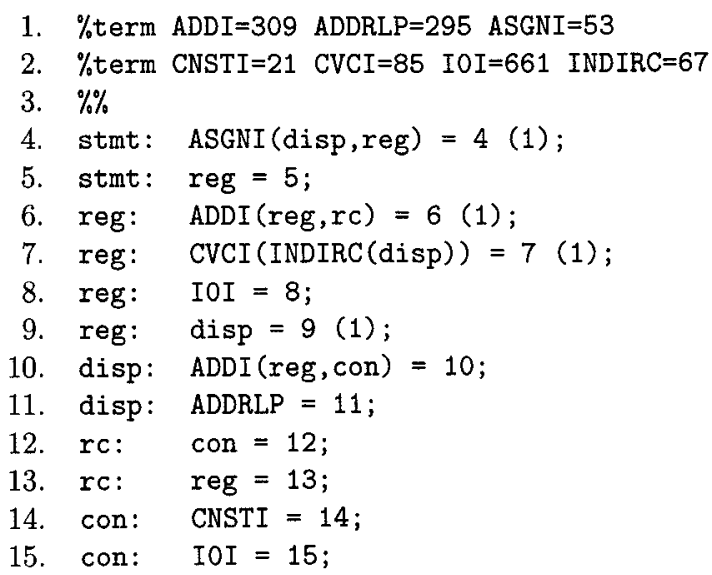

Fig. 2. Sample burg specification.

Each rule has a unique, positive external rule number, which comes after the pattern and is preceded by an equal sign. As described below, external rule numbers are used to report the matching rule to a user-supplied semantic action routine. Rules end with an optional nonnegative, integer cost; omitted costs default to zero.

Figure 2 shows a fragment of a burg specification for the VAX. This example uses uppercase for terminals and lowercase for nonterminals. Lines 1-2 declare the operators and their external symbol numbers, and lines 4-15 give the rules. The external rule numbers correspond to the line numbers to simplify interpreting subsequent figures. In practice, these numbers are usually generated by a preprocessor that accepts a richer form of specification (e.g., including YACC-style semantic actions) and that emits a burg specification [13]. Only the rules on lines 4, 6, 7, and 9 have nonzero costs. The rules on lines $5,9,12$, and 13 are chain rules. 
The operators in Figure 2 are some of the operators in lcc's intermediate language [10]. The operator names are formed by concatenating a generic operator name with a one-character type suffix like $\mathbf{C}, \mathbf{I}$, or $\mathbf{P}$, which denote character, integer, and pointer operations, respectively. The operators used in Figure 2 denote integer addition (ADDI), forming the address of a local variable (ADDRLP), integer assignment (ASGNI), an integer constant (CNSTI), "widening" a character to an integer (CVCI), the integer 0 (I0I), and fetching a character (INDIRC). The rules show that ADDI and ASGNI are binary; CVCI and INDIRC are unary; and ADDRLP, CNSTI, and IOI are leaves.

\section{MATCHING}

Both versions of burg generate functions that the client calls to label and reduce subject trees. The labeling function, label(p), makes a bottom-up, left-to-right pass over the subject tree $\mathbf{p}$ computing the rules that cover the tree with the minimum cost, if there is such a cover. Each node is labeled with $(M, C)$ to indicate that "the pattern associated with external rule $M$ matches the node with cost $C$."

Figure 3 shows the intermediate language tree for the assignment expression in the following $\mathrm{C}$ fragment:

$$
\{\text { int } \mathbf{i} \text {; char } \mathrm{c} ; \mathrm{i}=\mathrm{c}+4 \text {; }\}
$$

The left child of the ASGNI node computes the address of $\mathbf{i}$. The right child computes the address of $\mathbf{c}$, fetches the character, widens it to an integer, and adds 4 to the widened value, which the ASGNI assigns to i.

The other annotations in Figure 3. shows the results of labeling. $(M, C)$ denote labels from matches, and $[M, C]$ denote labels from chain rules. The rule from Figure 2 denoted by each $M$ is also shown. Each $C$ sums the costs of the nonterminals on the right-hand side and the cost of the relevant pattern or chain rule. For example, the pattern in line 11 of Figure 2 matches the node ADDRLP $i$ with cost 0 , so the node is labeled with $(11,0)$. Since this pattern denotes a disp, the chain rule in line 9 applies with a cost of 0 for matching a disp plus 1 for the chain rule itself. Likewise, the chain rules in lines 5 and 13 apply because the chain rule in line 9 denotes a reg.

Patterns can specify subtrees beyond the immediate children. For example, the pattern in line 7 of Figure 2 refers to the grandchild of the CVCI node. No separate pattern matches the INDIRC node, but line 7's pattern covers that node. The cost is the cost of matching the ADDRLP $i$ as a disp, which is rule 11, plus 1.

Nodes are annotated with $(M, C)$ only if $C$ is less than all previous matches for the nonterminal on the left-hand side of rule $M$. For example, the ADDI node matches the disp pattern in line 10 of Figure 2, which means that it also matches all rules with disp alone on the right-hand side, namely, line 9. By transitivity, it also matches the chain rules in lines 5 and 13 . But all three of these chain rules yield cost 2 , which is not better than previous matches for those nonterminals.

Once labeled, a subject tree is reduced by traversing it from the top down 


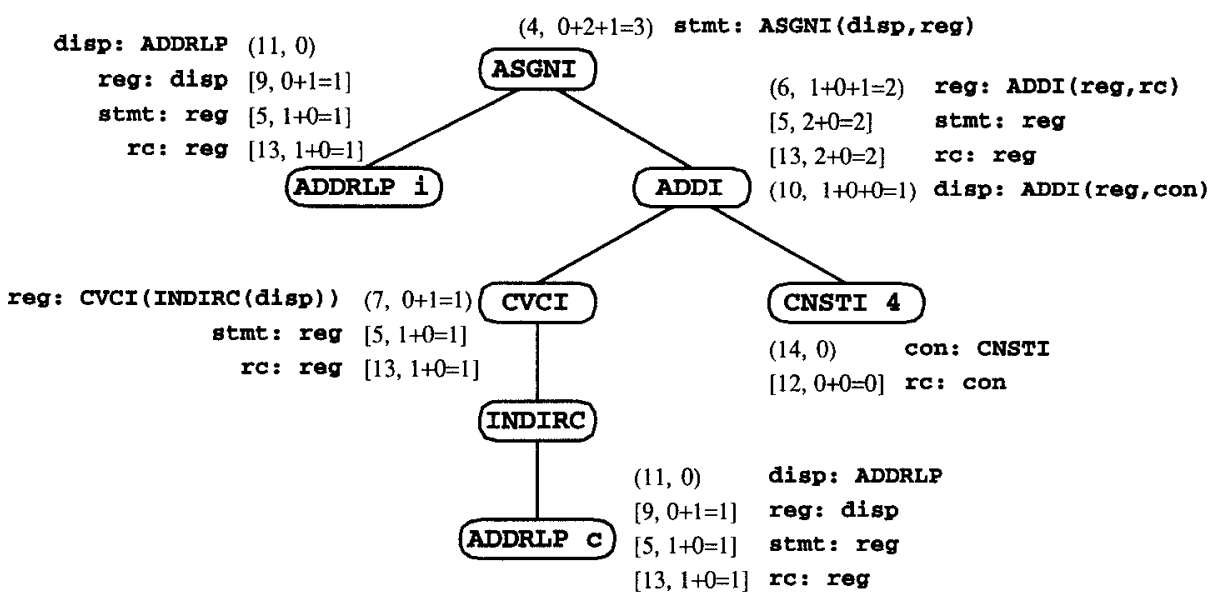

Fig. 3. Intermediate language tree for $\mathbf{i}=\mathbf{c}+\mathbf{4}$.

and by performing appropriate semantic actions, such as generating and emitting code. Reducers are supplied by clients, but burg generates functions that assist in these traversals, for example, one function that returns $M$ and another that identifies subtrees for recursive visits. Reference [13] elaborates.

burg does all DP at compile-compile time and annotates each node with a single, integral state number, which encodes all of the information concerning matches and costs. iburg does the DP at compile time and annotates nodes with data equivalent to $(M, C)$. Its "state numbers" are really pointers to records that hold these data.

Both versions of burg generate an implementation of label that accesses node fields via client-supplied macros or functions and uses the nonrecursive function state to identify matches:

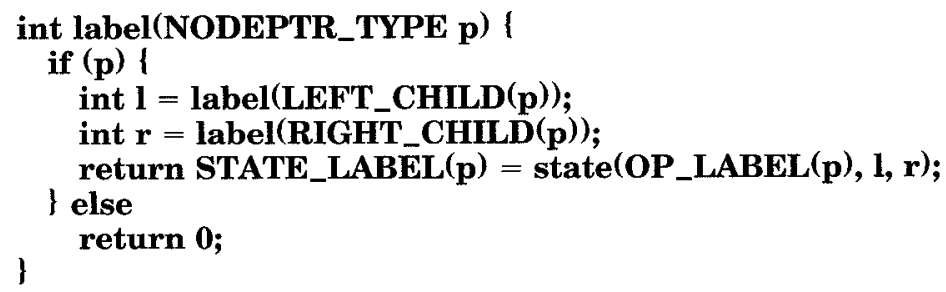

NODEPTR_TYPE is a typedef or macro that defines the data type of nodes. OP_LABEL, LEFT_CHILD, and RIGHT_CHILD are macros or functions that return, respectively, a node's external symbol number, its left child, and its right child. STATE_LABEL is a macro that accesses a node's state number field.

state accepts an external symbol number for a node and the state numbers for the node's left and right children. It returns the state number to assign to that node. For unary operators and leaves, it ignores the last one or two arguments, respectively. 


\section{IMPLEMENTATION}

iburg generates a state function that uses a straightforward implementation of tree pattern matching [7]. It generates hard code instead of tables. Its "state numbers" are pointers to state records, which hold vectors of the ( $M$, $C$ ) values for successful matches. The state record for the specification in Figure 2 is the following:

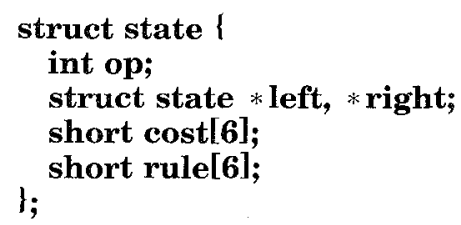

iburg also generates integer codes for the nonterminals, which index the cost and rule vectors:

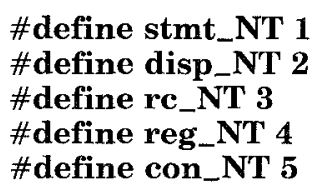

By convention, the start nonterminal has the value 1 .

State records are cleared when allocated, and external rule numbers are positive. Thus, a nonzero value for $\mathbf{p} \rightarrow \mathbf{r u l e}[X]$ indicates that $\mathbf{p}$ 's node matched a rule that defines nonterminal $X$.

Figure 4 shows the implementation of state and gives the cases that are contributed by Figure 2's lines 6, 7, 10, and 11. state allocates and initializes a new state record and switches on the external symbol number to begin matching. Each nonleaf case is one or more if statements that test for a match by consulting the state records of descendants. The switch by itself does all the necessary testing for leaves.

If a match succeeds, the resulting cost is computed, and record is called with the pointer to the state record, the code for the matching nonterminal, the cost, and the matching external rule number:

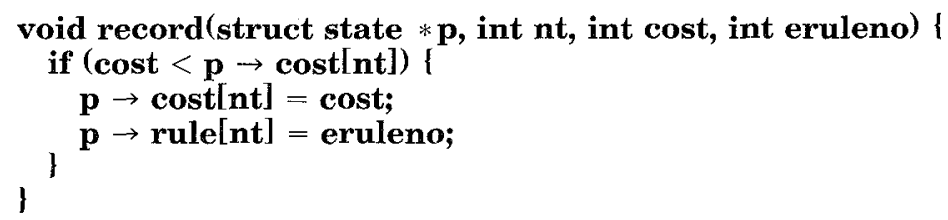

The match is recorded only if its cost is less than previous matches. The elements of the cost vector are initialized with 32767 to represent infinite cost, so the first match is always recorded.

The first call to record is for the match itself; the other calls are for chain rules. For example, the second if statement in the ADDI case tests whether p's node matches the pattern in line 10 . If it does, the first call to record records that the node matches a disp. The chain rule in line 9 says that a 


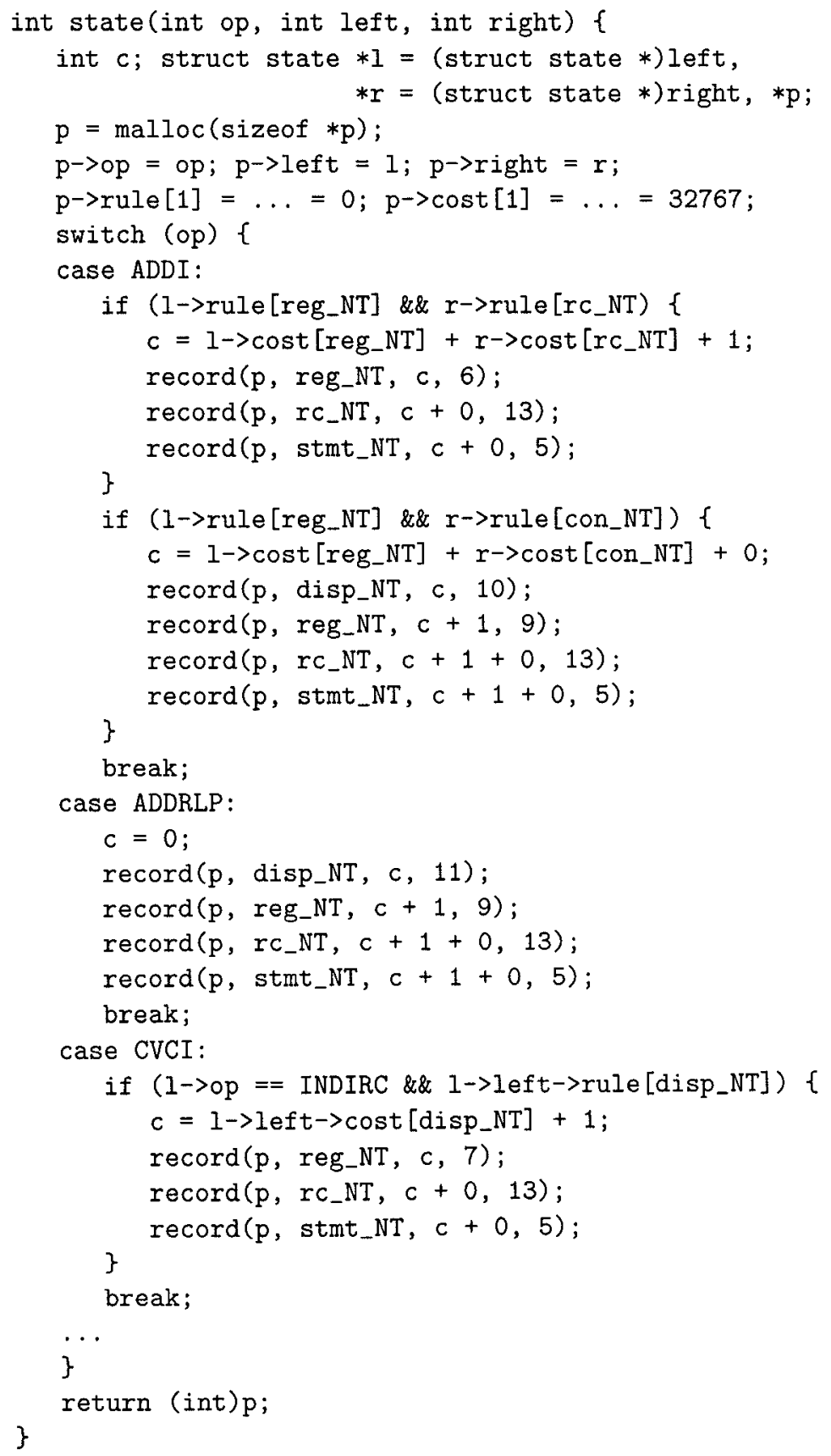

Fig. 4. Implementation of state. 
node matching a disp also matches a reg with an additional cost of 1 , which gives rise to the second record call. Likewise, the last two calls to record are due to the chain rules in lines 5 and 13 , which say that a node matching a reg also matches a stmt and an rc, both with an additional cost of 0 . In general, there is a call to record for the transitive closure of all chain rules that reach the nonterminal defined by the match.

\section{IMPROVEMENTS}

The generated matcher described in the previous section is practical for many code-generation applications, and the generator itself is easy to implement. Students have replicated the version that emits the code shown in Figure 4 in a couple of weeks. iburg implements, however, several simple improvements that make the generated matchers smaller and faster. Even with the improvements below, iburg takes only 642 lines of Icon [14].

The short elements of the rule vector can accommodate any external rule number, but many nonterminals are defined by only a few rules. For example, only lines 10 and 11 in Figure 2 define disp, so only two bits are needed to record one of the two positive values. Definitions can be mapped into a compact range of integers and stored in minimum space in state records as bit fields, as in the following example:

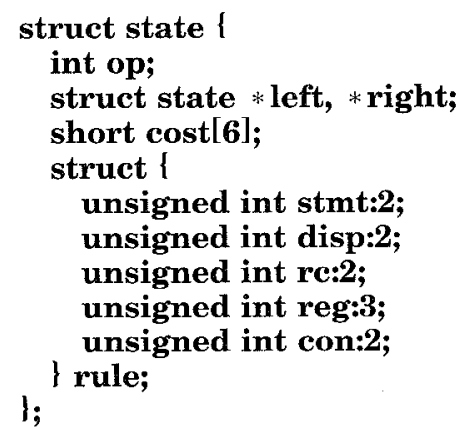

External rule numbers for matches are retrieved by calling rule with a state number and a goal nonterminal [13]. iburg generates an implementation of rule that uses tables to map the integers in the compact representation to external rule numbers, as in the following example:

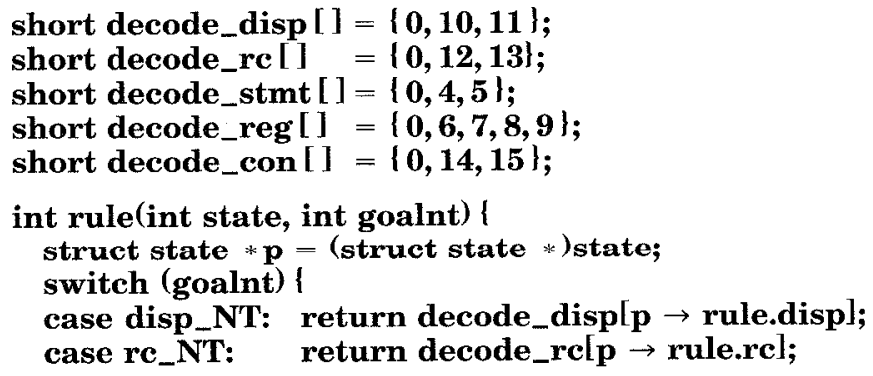

ACM Letters on Programming Languages and Systems, Vol. 1, No. 3, September 1992. 


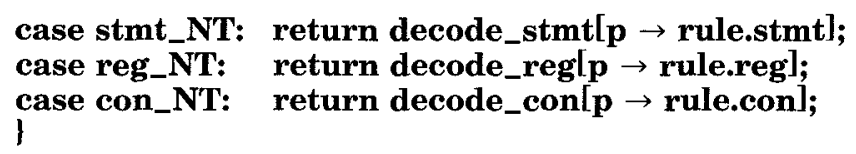

Packed rule numbers cannot be subscripted, so record and the code that tests for a match must be changed. This scheme can save much space for large specifications. For example, the VAX specification has 47 nonterminals, and the encoding scheme reduces the size of its rule vector from 96 to 16 bytes.

Packing rule numbers can also save time: It takes longer to read, write, and decode packed rule numbers, but the smaller structure can be initialized much faster, with a single structure copy. The original VAX matcher initialized rule with 47 assignments; a structure copy would have been slower. With packed fields, 47 assignments would be slower yet, but a 16-byte structure copy beats the original 47 assignments by a margin that swamps the other costs of using packed fields.

Initialization costs can be reduced further still: All costs much be set, but only the rule field for the start symbol needs initialization. The rule fields are read in only two places: the rule function above, and the tests for a match. The rule function is called during a top-down tree traversal, which always begins with the start symbol as the goal nonterminal. If it finds the initializer's zero rule number, then the tree failed to match, and no more fields should be examined anyway. The match tests require no rule initialization at all. They read the rule fields of descendants; if they read garbage, then the descendants failed to match, and their costs will be infinite, which will prevent recording a false match. With this improved initializer, packing rule numbers no longer saves time, but it still saves space, and the time cost is so small that it could not be measured.

record can also be improved. If the cost test in record fails, the tests in the calls to record that implement its chain rules must fail too, because costs increase monotonically. These calls can be avoided if the cost test fails. Inlining record accommodates both this improvement and packed rules. For example, the second if statement in the ADDI case in Figure 4 becomes the following:

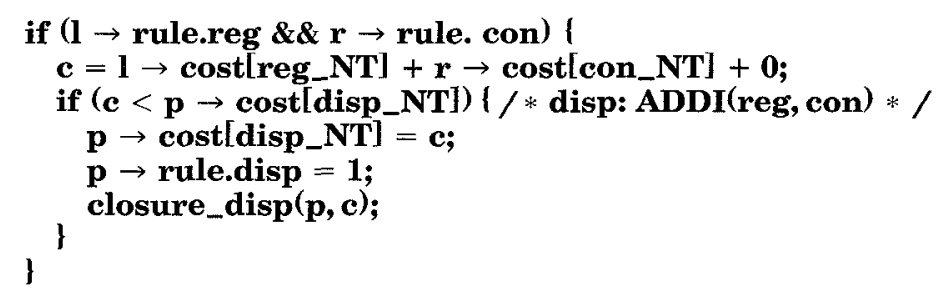

$\mathbf{p} \rightarrow$ rule.disp is set to 1 because decode_disp above maps 1 to external rule 10 .

This code also shows a more compact approach to handling chain rules. For each nonterminal $X$ that can be reached via chain rules, iburg generates 
closure_ $X$, which records the chain rule match if its cost is better than previous matches and, if applicable, calls another closure function. For example, the closure function for disp is the following:

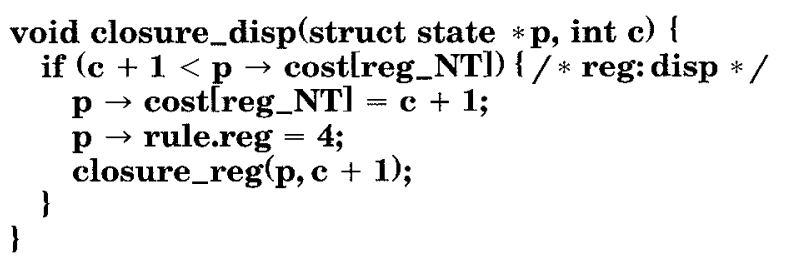

The incoming cost, $\mathbf{c}$, is the cost of matching the right-hand side of the chain rule. This cost plus the cost of the chain rule itself, for example, 1 for line 9's reg: disp, is the cost of this application of the chain rule, and this sum is passed to the next closure function. closure_reg handles both chain rules for reg (lines 5 and 13):

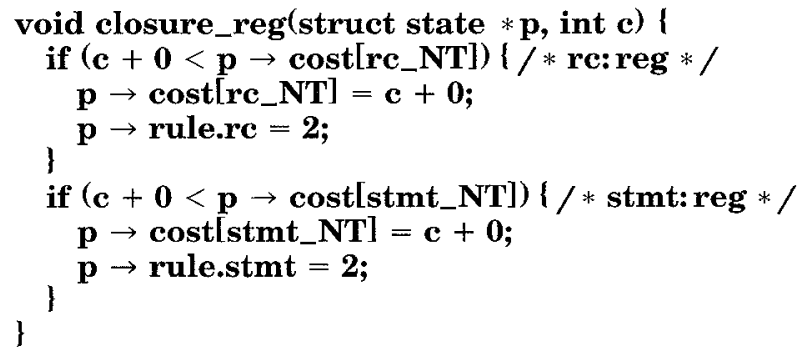

The final improvement saves times for leaves, which abound in subject trees from code generators. The computation and encoding of all of the state record data about matches at compile-compile time are complicated [18]. Leaves, however, always match, and the contents of the state record are easily computed by simulating the effect of the assignments and closure function calls shown above. The state records for leaves can thus be allocated and initialized at compile-compile time; for example, the ADDRLP case in Figure 4 becomes the following:

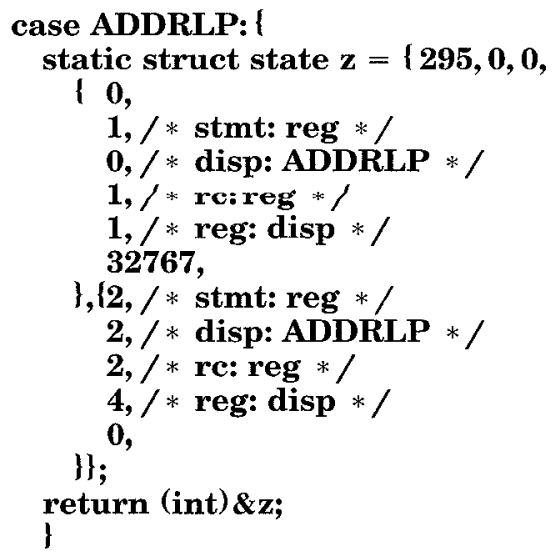


Table I. Improvements

\begin{tabular}{ccccl}
\hline $\begin{array}{c}\text { iburg } \\
\text { size }\end{array}$ & $\begin{array}{c}\text { Matcher } \\
\text { size }\end{array}$ & $\begin{array}{c}\text { lec } \\
\text { time }\end{array}$ & $\begin{array}{c}\text { Matcher } \\
\text { time }\end{array}$ & \multicolumn{1}{c}{ Version } \\
\hline 566 & 240,140 & 2.5 & .69 & Original untuned version \\
580 & 56,304 & 2.4 & .59 & Inline record; add closure routines \\
580 & 56,120 & 2.4 & .59 & Initialize only one element of rule \\
616 & 58,760 & 2.2 & .39 & Precompute leaf states \\
642 & 66,040 & 2.2 & .39 & Pack rule numbers \\
\hline
\end{tabular}

The first three values initialize the op, left, and right fields of the state structure. The two brace-enclosed initializers given the cost and rule values, respectively. The code at the beginning of state (see Figure 4) that allocates and initializes a state record is not needed for leaves, so it is protected by a test that excludes leaf ops.

Table I traces the addition of each improvement above. The first column shows the number of lines of Icon in iburg and helps quantify implementation cost. The second column shows the number of object bytes in the resulting matcher. The third column times lec in a typical cross-compilation for the VAX on a MIPS processor. The fourth column shows the time spent in the state and rule routines. All times are in seconds. Specifications for RISC machines would show smaller improvements.

Closure routines save so much space because they implement chain rules in one place rather than in multiple record runs. The initialization improvement could not be measured in this trial, but it is trivial to implement and must save something. On the other hand, packing rule numbers must have cost some time, but the cost appears small, and it cuts the size of the state structure by almost half.

Two proposed improvements proved uneconomical. First, the closure routines were inlined; measurements showed that the matcher was faster, but it was larger than even the initial version above. Independently, the closure routines were recoded to avoid tail recursion, but no speedup was measured. The recoding replaced each closure routine with a case in a switch statement, and the switch bound check added unnecessary overhead; so it is possible that a compiler implementation of tail recursion could do better, though large speedups seem unlikely.

Much of the processing done by iburg is straightforward. For example, parsing the input and writing the output account for 181 and 159 lines, respectively, in the 642-line final version. By way of comparison, a new version of iburg written in $\mathrm{C}$ is 950 lines, and a burg processor is 5100 lines of C [18].

\section{DISCUSSION}

iburg was built to test early versions of what evolved into burg's specification language and interface. Initial tests used Twig and a Twig preprocessor, but Twig produced incorrect matchers for large CISC grammars. The error proved hard to find, so Twig was abandoned and iburg was written. The 
Table II. Times for Compiling C Programs in the SPEC Benchmarks

\begin{tabular}{lcc}
\hline Benchmark & iburg & iburg \\
\hline 001.gce & 90.2 & 77.9 \\
008.espresso & 28.3 & 24.6 \\
022.li & 8.9 & 8.0 \\
023.eqntott & 5.6 & 4.9 \\
\hline
\end{tabular}

initial version was completed in two days with 200 lines of Icon. The final, student-proof version with full burg-compatible debugging support is 642 lines.

Matchers generated by iburg are slower than those from burg. Table II shows the times for compiling the C programs in the SPEC benchmarks [19] with two versions of lcc. These times are for running only the compiler proper; preprocessing, assembly, and linking time are not included. The compilations were done on an IRIS $4 \mathrm{D} / 220 \mathrm{GTX}$ with $32 \mathrm{MB}$ running IRIX 3.3.2, and the times are elapsed time in seconds and are the lowest elapsed times over several runs on a lightly loaded machine. All reported runs achieved at least 96 percent utilization [i.e., the ratio of times (user + system)/elapsed $\geq 0.96]$.

The differences in compilation times are due entirely to differences in the performance of the two matchers. Profiling shows that the execution time of iburg's rule is nearly identical to burg's rule. On these inputs, iburg's matcher accounts for 8.5-12.4 percent of the execution time, whereas burg's accounts for only 1.1-2.0 percent, making burg roughly 6-12 times faster.

Comparable figures for Twig are unavailable because it did not correctly process large grammars, but before work with Twig was abandoned, a few measurements were taken. Using a nearly complete VAX grammar, lcc compiled one 2000-line module in $20.71 \mathrm{~s}$ using a Twig matcher and $5.35 \mathrm{~s}$ using a matcher from the initial iburg; it spent $15.64 \mathrm{~s}$ in the Twig matcher and $0.85 \mathrm{~s}$ in the iburg matcher. Using a partial MIPS grammar, lec compiled the module in $9.19 \mathrm{~s}$ using a Twig matcher and $4.54 \mathrm{~s}$ using a matcher from the initial iburg; it spent $4.04 \mathrm{~s}$ in the Twig matcher and $0.16 \mathrm{~s}$ in the iburg matcher. Both versions of lcc used a naive emitter that was slowed by complex grammars, which is why the VAX compiler was so much slower. The figures in this paragraph are useful for comparing Twig with iburg, but the naive emitter makes them useless for comparisons with anything else.

A disadvantage of BURS matchers is that the costs must be constant because the DP is done at compile-compile time. Costs in Twig specifications, however, can involve arbitrary computation and can depend on context. For example, the pattern

\section{ASGNI(disp, CNSTI)}

specifies a clear instruction if the constant is 0 . Twig's cost computations can inspect the subject tree and return a cost of, say, 1 if the constant is 0 and infinity otherwise. 
BURS specifications can handle this kind of context sensitivity with additional operators that identify the special cases. For example, before calling state, lcc's labeling pass changes CNSTI to I0I if the constant is 0. Thus,

\section{ASGNI(disp, I0I)}

specifies a clear instruction.

Most context-sensitive cases that arise in code generation, even for CISC machines, can be handled similarly, perhaps with a few additional rules. For example, recognizing and using the VAX's indexed addressing mode takes 12 rules in lcc's specification. iburg could easily be extended so that predicates could be specified and tested during matching, much like BEG's conditions [7].

iburg can be useful during development. The generated state and label functions are easy to read and to debug. Indeed, they mirror their specification in the same way that the code for a recursive-descent parser mirrors its LL(1) grammar. This attribute has made iburg ideal for teaching. It has been used in a course that previously used Twig, but students prefer iburg. When students make their inevitable mistakes with a table-driven matcher like Twig's or burg's, only inscrutable numbers from the table are available from the debugger. When they make mistakes with iburg, each node explicitly records the matching rules and costs for each nonterminal, so users can easily compare the matcher's actual operation with their expectations.

\section{ACKNOWLEDGMENTS}

Section 2 borrows from [13], parts of which were written by Robert Henry. The $\mathrm{C}$ version of iburg is available for anonymous ftp from ftp.cs.princeton.edu in pub.

\section{AUTHORS' NOTE}

Section 5 notes that the cost tests make it unnecessary to initialize most rule fields. BEG [7] carried this observation one step further. Tests like the outer if statement in the improved ADDI case in Section 5 need not test rule fields at all; the cost tests suffice. Such if statements are necessary only if there are embedded terminals to test, like the INDIRC in the rule on line 7 of Figure 2. This improvement has been added to iburg. Trials could not quantify an improvement, but it probably saves something, and it is easier to read.

\section{REFERENCES}

1. AHO, A. V., AND CoRASICK, M. J. Efficient string matching: An aid to bibliographic search. Commun. ACM 18, 6 (June 1975), 333-340.

2. AHo, A. V., AND Johnson, S. C. Optimal code generation for expression trees. J. ACM 23, 3 (July 1976), 488-501.

3. AHo, A. V., GaNapathi, M., ANd T.JANG, S. W. K. Code generation using tree matching and dynamic programming. ACM Trans. Program. Lang. Syst. 11, 4 (Oct. 1989), 491-516.

4. Aho, A. V., Sethi, R., And Ullman, J. D. Compilers: Principles, Techniques, and Tools. Addison-Wesley, Reading, Mass., 1986. 
5. Balachandran, A., Dhamdhere, D. M., and Biswas, S. Efficient retargetable code generation using bottom-up tree pattern matching. J. Comput. Lang. 15, 3 (1990), 127-140.

6. Chase, D. R. An improvement to bottom-up tree pattern matching. In Conference Record of the ACM Symposium on Principles of Programming Languages (Munich, Germany, Jan. 21-23, 1987). ACM, New York, 168-177.

7. Emmelmann, H., Schröer, F.-W., ANd Landwehr, R. BEG-A generator for efficient back ends. In Proceedings of the SIGPLAN 89 Conference on Programming Language Design and Implementation. SIGPLAN Not. (ACM) 24, 7 (July 1989), 227-237.

8. Ferdinand, C., Seidl, H., and Wilhelm, R. Tree automata for code selection. In Code Generation-Concepts, Tools. Techniques, Proceedings of the International Workshop on Code Generation (Dagstuhl, Germany), R. Giegerich and S. L. Graham, Eds. Springer-Verlag, New York, 1991, 30-50.

9. Fraser, C. W. A language for writing code generators. In Proceedings of the SIGPLAN 89 Conference on Programming Language Design and Implementation. SIGPLAN Not. (ACM) 24, 7 (July 1989), 238-245.

10. Fraser, C. W., And Hanson, D. R. A code generation interface for ANSI C. Softw. Pract. Exper. 21, 9 (Sept. 1991), 963-988.

11. Fraser, C. W., ANd Hanson, D. R. A retargetable compiler for ANSI C. SIGPLAN Not. (ACM) 26,10 (Oct. 1991), 29-43.

12. Fraser, C. W., and Henry, R. R. Hard-coding bottom-up code generation tables to save time and space. Softw. Pract. Exper. 21, 1 (Jan. 1991), 1-12.

13. Fraser, C. W., Henry, R. R., and Proebsting, T. A. BURG-Fast optimal instruction selection and tree parsing. SIGPLAN Not. (ACM) 27, 4 (Apr. 1992), 68-76.

14. GRIswold, R. E., AND GRISwold, M. T. The Icon Programming Language. 2nd ed. PrenticeHall, Englewood Cliffs, N.J., 1990.

15. Hoffman, C. M., And O'Donnell, M. J. Pattern matching in trees. J. ACM 29, 1 (Jan. 1982), 68-95.

16. PeleGri-Llopart, E. Tree transformation in compiler systems. Ph.D. thesis, Computer Science Division, Dept. of Electrical Engineering and Computer Science, Univ. of California, Berkeley, Calif., Dec. 1987.

17. Pelegri-Llopart, E., ANd Graham, S. L. Optimal code generation for expression trees: An application of BURS theory. In Conference Record of the ACM Symposium on Principles of Programming Languages (San Diego, Calif., Jan. 13-15, 1988). ACM, New York, pp. 294-308.

18. Proebsting, T. A. Simple and efficient BURS table generation. In Proceedings of the SIGPLAN 92 Conference on Programming Language Design and Implementation. SIGPLAN Not. (ACM) 27, 6 (June 1992), 331-340.

19. Standards Performance Evaluation Corp. SPEC Benchmark Suite Release 1.o. Standards Performance Evaluation Corp., Oct. 1989.

Received October 1992; revised and accepted January 1993 